$\mathrm{e}^{+} \mathrm{e}^{-}$Collisions from Phi to Psi 2013 (PHIPSI2013)

International Journal of Modern Physics: Conference Series

Vol. 35 (2014) 1460463 (3 pages)

(C) The Authors

DOI: $10.1142 /$ S2010194514604633

\title{
DETERMINATION OF THE MAGNETIC DIPOLE MOMENT OF THE RHO MESON
}

\author{
G. TOLEDO SÁNCHEZ* and D. GARCÍA GUDIÑO ${ }^{\dagger}$ \\ Instituto de Física, Universidad Nacional Autónoma de México, \\ AP 20-364, México D.F. 01000, México. \\ *toledo@fisica.unam.mx \\ $\dagger$ david.garcia@fisica.unam.mx
}

Published 18 December 2014

\begin{abstract}
We determine the magnetic dipole moment of the rho meson using preliminary data from the BaBar Collaboration for the $e^{+} e^{-} \rightarrow \pi^{+} \pi^{-} 2 \pi^{0}$ process, in the center of mass energy range from 0.9 to $2.2 \mathrm{GeV}$. We describe the $\gamma^{*} \rightarrow 4 \pi$ vertex using a vector meson dominance model, including all intermediate resonance contributions. We find that $\mu_{\rho}=2.1 \pm 0.5\left[e / 2 m_{\rho}\right]$.
\end{abstract}

Keywords: Vector mesons; electromagnetic properties.

PACS Numbers: 13.40.Gp, 12.40.Vv, 11.10.St, 13.66.Bc

To date, there is no measurement of the magnetic dipole moment (MDM) of any vector meson. ${ }^{1}$ This fact is related to their extremely short lifetimes $\left(\approx 10^{-23} \mathrm{~s}\right)$, which prevents experimentalists to apply standard MDM measurement techniques. Alternatives to determine the MDM, by indirect means, invoke the fact that the radiation emitted from the vector meson carries information of its electromagnetic structure $^{2}$ and thus, provided the dominant electric radiation is known, the subleading MDM effect can be identified.

We present ${ }^{3}$ the first determination of a vector meson MDM, namely the MDM of the $\rho$ meson, obtained from the $e^{+} e^{-} \rightarrow \pi^{+} \pi^{-} 2 \pi^{0}$ process, using preliminary data from the BaBar Collaboration. ${ }^{4}$ The $\gamma^{*} \rightarrow 4 \pi$ vertex is modeled in the vector meson dominance (VMD) approach. This process is sensitive to the channel where the pions are produced in pairs through $\rho$ meson resonant states, which is related to the $\rho$ electromagnetic vertex. The MDM is determined by fitting the experimental cross section data, while using other observables to fix all the remaining parameters.

For a vector particle $(\mathrm{V})$, the vertex for the process $V\left(q_{1}, \epsilon\right) \rightarrow V\left(q_{2}, \eta\right) \gamma(q)$ is defined from the electromagnetic current $<V\left(q_{2}\right)\left|J_{E M}^{\mu}(0)\right| V\left(q_{1}\right)>\equiv \eta_{\nu}^{\dagger} \epsilon_{\lambda} \Gamma^{\mu \nu \lambda}$,

This is an Open Access article published by World Scientific Publishing Company. It is distributed under the terms of the Creative Commons Attribution 3.0 (CC-BY) License. Further distribution of this work is permitted, provided the original work is properly cited. 
where $q_{i}$ are the momenta and $\epsilon$ and $\eta$ are the corresponding polarization tensors. The CP conserving electromagnetic vertex $\Gamma^{\mu \nu \lambda}$ can be decomposed into the following Lorentz structures

$$
\begin{aligned}
\Gamma^{\mu \nu \lambda}= & \alpha\left(q^{2}\right) g^{\nu \lambda}\left(q_{1}+q_{2}\right)^{\mu}+\beta\left(q^{2}\right)\left(g^{\mu \nu} q^{\lambda}-g^{\mu \lambda} q^{\nu}\right) \\
& -\gamma\left(q^{2}\right)\left(q_{1}+q_{2}\right)^{\mu} q^{\nu} q^{\lambda} .
\end{aligned}
$$

The electromagnetic form factors $\alpha\left(q^{2}\right), \beta\left(q^{2}\right)$ and $\gamma\left(q^{2}\right)$ dependence on $q^{2}$ is modeled by VMD and, in the static limit, are related to the electromagnetic multipoles as follows: $|\mathcal{Q}|=\alpha(0)$ is the electric charge (in $e$ units), $|\vec{\mu}|=\beta(0) \equiv 1+\kappa+\lambda$ is the magnetic dipole moment (in $e / 2 M_{V}$ units) and the electric quadrupole is $\left|X_{E}\right|=\kappa-\gamma(0) M_{V}^{2} \equiv \kappa-\lambda$ (in $e / M_{V}^{2}$ units), where we have included the relation to the parameters $\kappa$ and $\lambda$ which are also of common use in the literature to refer to the electromagnetic multipoles of spin- 1 particles. ${ }^{5,6}$ For instance, at tree level, the standard model gauge structure predicts for the $W$ gauge boson to have $\alpha(0)=1$, $\beta(0)=2$ and $\gamma(0)=0(\kappa=1$ and $\lambda=0)$, corresponding to $|\mathcal{Q}|=1,|\vec{\mu}|=2$ and $\left|X_{E}\right|=1$. These values are usually taken as a reference for vector mesons.

The predictions for the $\operatorname{MDM} \rho$ meson lay in the region from 1.9 to $3 e / 2 M_{\rho}$.

The $e^{+} e^{-} \rightarrow \pi^{+} \pi^{-} 2 \pi^{0}$ process has been measured by several experiments in a direct way at low energies, ${ }^{7}$ and preliminary data is available from the BaBar collaboration $^{4}$ in wider range.

The description we follow for the hadronic part is based on VMD. The channels here considered include the exchange of the $\pi, \omega, a_{1}, \sigma$ (and $f(980)$ ), $\rho$ and $\rho^{\prime}(1450)$ mesons. The energy range to be described goes from threshold up to 2.2 $\mathrm{GeV}$. Thus, we are left with seven generic channels, each one accounting for several specific diagrams, corresponding to the allowed permutations of the momenta due to Bose-Einstein symmetry and charge conjugation. We take the same structure and coupling for the case when the $\rho^{\prime}$ is included, this assumption has been found to be appealing. ${ }^{8}$ The vector meson propagator takes the complex mass form. The only free parameters are those involved in the electromagnetic vertex $\Gamma^{\nu \beta \gamma}$. Namely, the $\beta$ and $\gamma$ parameters, since the electric charge is fixed.

The electromagnetic structure of the $\rho$ meson as a function of the momentum is accomplished by the inclusion of the $\rho$ and $\rho^{\prime}$ resonances couplings to the photon, with a relative phase of $180^{\circ}$.

In Figure 1 we show the total cross section data with a $10 \%$ systematic error bars, ${ }^{4}$ and the fit corresponding to $\beta$ as a free parameter and $\gamma=0$. The fit considering $\beta$ and $\gamma$ as free parameters favors the same $\beta$ and restricts $\gamma$ to be in the range $(-1.1,0.1)$. That is, the $\beta$ parameter accounts for the global description, while the $\gamma$ contribution enters at the end region. To determine the cross section error bars, we have taken into account the combined uncertainties coming from the couplings of the different channels, assumed as no correlated. We also explored the role of the model assumption regarding the $\rho^{\prime}$ triple boson vertex, the global combination of couplings and mass was found to be consistent with data for upto a $10 \%$ deviation from the combination for the $\rho$. We determine the $\beta$ parameter error bar considering 


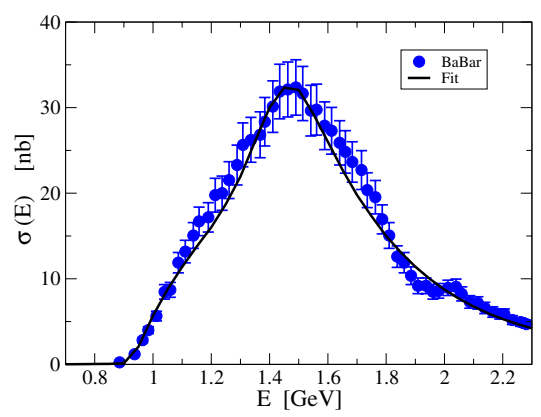

Fig. 1. Fit to the BaBar data for the total cross section $e^{+} e^{-} \rightarrow \pi^{+} \pi^{-} 2 \pi^{0}$. We use the MDM as the only free parameter, while the other involved parameters are fixed from other observables.

it as the responsible of the total uncertainties. In addition, to account for the model dependence, we have added a $20 \%$ error (added in quadrature). Thus, corresponding to a $\mathrm{MDM}$

$$
\mu_{\rho}=2.1 \pm 0.5\left[\frac{e}{2 m_{\rho}}\right]
$$

In summary, we have determined the magnetic dipole moment of the $\rho$ meson, by studying its effect in the $e^{+} e^{-} \rightarrow \pi^{+} \pi^{-} 2 \pi^{0}$ cross section, in the VMD approach. The channel that contains the electromagnetic vector meson vertex becomes relevant for energies between 1.5 and $2.2 \mathrm{GeV}$, while the remaining channels are always subdominant. We have found that the best fit to the BaBar data implies a value for the MDM of the $\rho$ meson of $\mu_{\rho}=2.1 \pm 0.5\left[\frac{e}{2 m_{\rho}}\right]$.

The quoted error bar takes into account the uncertainties coming form the couplings of the different channels and model assumptions, definite data on this process and detailed information on the $\rho^{\prime}$ meson will be very useful for a more refined analysis.

\section{Acknowledgments}

We acknowledge the support of CONACyT-México under grant 128534, and DGAPA-PAPIIT UNAM, under grants IB101012 and IN106913.

\section{References}

1. J. Beringer et al. (Particle Data Group), Phys. Rev. D 86, 010001(2012).

2. V. I. Zakharov, L. A. Kondratyuk and L. A. Ponomarev, Sov. J. of Nucl. Phys. 8 456(1969).

3. D. Garcia Gudino and G. Toledo Sanchez, arXiv: 1305.6345 [hep-ph].

4. V. P. Druzhinin, arXiv: 0710.3455 [hep-ex].

5. K. Hagiwara, R. D. Peccei, D. Zeppenfeld and K. Hikasa, Nucl. Phys. B282, 253 (1987).

6. J. F. Nieves and P. B. Pal, Phys. Rev. D 553118 (1997).

7. M. N. Achasov et al. (SND Collaboration) J. Exp. Theor. Phys. 96789 (2003); J. Exp. Theor. Phys. 109 379(2009).

8. H. Czyz, J. H. Kuhn and A. Wapienik, Phys. Rev. D 77, 114005 (2008) 